Recherches en didactique des langues et des cultures

Les cahiers de l'Acedle

14-2 | 2017

Dialogisme et discours en situations didactiques

\title{
Le cours magistral de littérature : un dialogisme digressif et polyphonique
}

François Raviez et Jean-Marc Mangiante

\section{OpenEdition}

Journals

Édition électronique

URL : http://journals.openedition.org/rdlc/1858

DOI : $10.4000 /$ rdlc. 1858

ISSN : 1958-5772

Éditeur

ACEDLE

Référence électronique

François Raviez et Jean-Marc Mangiante, « Le cours magistral de littérature : un dialogisme digressif et polyphonique », Recherches en didactique des langues et des cultures [En ligne], 14-2 | 2017, mis en ligne le 15 juin 2017, consulté le 19 avril 2019. URL : http://journals.openedition.org/rdlc/1858; DOI : $10.4000 /$ rdlc. 1858

Ce document a été généré automatiquement le 19 avril 2019

\section{(c) (1) $\Theta$}

Recherches en didactique des langues et des cultures is licensed under a Creative Commons AttributionNonCommercial-NoDerivatives 4.0 International License 


\title{
Le cours magistral de littérature : un dialogisme digressif et polyphonique
}

\author{
François Raviez et Jean-Marc Mangiante
}

\section{Introduction}

1 Comme tout discours de transmission des connaissances, le cours magistral relève d'un double dialogisme (Bakhtine, 1938/1978): interlocutif dans sa prise en compte des réactions des destinataires - en l'occurrence les étudiants susceptibles de réagir et d'interagir avec l'enseignant - et interdiscursif dans sa convocation de multiples discours de référence intégrés aux discours de l'enseignant (Mangiante et Parpette, 2011).

2 Ce dialogisme bi-polaire s'inscrit dans un cours magistral qui possède des caractéristiques propres parmi lesquelles la digression constitue la principale en ce sens qu'elle influence directement la relation entre l'enseignant et les étudiants.

3 Cet article se propose de définir et de décrire le dialogisme particulier du cours magistral de littérature en le reliant à la multiplicité des voix convoquées par l'enseignant et à sa propension à développer une forme spécifique de digression qui ne constitue pas un détournement rhétorique de la parole enseignante vers des énoncés latéraux, mais un approfondissement parfois plus important que les énoncés notionnels définis par ce dernier (Raviez, 2014).

4 La démarche s'appuiera sur des exemples concrets d'extraits de cours magistraux analysés et exploités pédagogiquement en cours de Français sur Objectif Universitaire (désormais FOU), de Licence de Lettres Modernes, et destinés à la formation linguistique et méthodologique des étudiants allophones et natifs (Mangiante \& Raviez, 2015). Ces extraits sont issus du corpus de cours enregistrés dans la filière de Licence de Lettres Modernes de l'université d'Artois et constitué par l'équipe de recherche du laboratoire Grammatica qui travaille sur l'analyse des discours universitaires et la didactique du 
Français sur Objectif Universitaire. Une partie du corpus a été utilisé dans le DVD d'accompagnement de l'ouvrage Réussir ses études littéraires en français (Mangiante \& Raviez, 2015).

\section{La réception du cours magistral de littérature : les conditions de la compréhension orale en situation dialogique}

Un certain nombre de questions se posent à l'enseignant de littérature face à des étudiants dont il entend tenir compte des réactions. Comment l'étudiant perçoit-il un cours de littérature? La question en amène d'autres : quelle trace écrite en garde-t-il ? Qu'en retire-t-il sur les plans intellectuel, méthodologique, voire personnel, une fois le cours terminé? Comment l'utilisera-t-il ? Comment intégrera-t-il les nombreuses références et voix différentes, voire discordantes parfois, qui émaillent le discours de l'enseignant ? En fera-t-il une synthèse, une analyse comparative, etc.?

Toute réponse suppose que l'on envisage d'abord les déplacements de la parole : de celle du professeur (associée à un corps, une voix, tout autant qu'à une personnalité, une culture, une compétence) à la fragmentation des notes prises en classe, mais aussi à ce qui en perdure dans la mémoire, à ce que l'intelligence en fera, à ce qui nourrira la sensibilité.

La parole du professeur connaît aussi, en amont, diverses métamorphoses : avant d'être oralisée devant la classe (c'est-à-dire jouée, performée, scénarisée), elle a été préparée, orchestrée, organisée. Le professeur s'est confronté à un auteur et, de la lecture à l'élaboration du cours, a fait entrer dans son propos tout ce qu'il a pu apprendre et comprendre de cet auteur. Il cherche maintenant à le bien faire entendre : à l'instant même où il prend la parole, se diffracte un savoir pluriel, un composé d'éléments objectifs (une date, une figure, une citation) et d'appréciations personnelles.

Le cours est nécessairement partiel: la pointe visible d'une culture et d'une herméneutique. Ainsi, des deux côtés du bureau il y a sélection, c'est-à-dire réduction, mais aussi répétition. Le cours redit, dans les mots du moment, ce que le professeur a noté devoir être dit : il cite tel auteur, mais aussi se cite lui-même, il reproduit devant la classe le travail antérieur d'appropriation d'une œuvre, il le transmet en usant d'images, de synonymes, d'équivalents qui se mêlent d'effets de rappels ou d'annonce, de conseils, de références, et quelquefois de commentaires privés. Ainsi, son cours relevant d'un dialogisme interdiscursif, l'enseignant multiplie les recours aux références - sources de son cours assorties de ses propres commentaires, apports, prises de position personnelles dans cette mise en concurrence ou en complémentarité des différentes contributions à son argumentation didactique.

9 La difficulté pour l'étudiant dans sa démarche de compréhension du cours est d'abord le repérage et l'identification des références ainsi que les liens qui les unissent. En effet les références ne peuvent s'appréhender indépendamment les unes des autres, elles ne peuvent être comprises que selon leur appartenance à un tout, au raisonnement global et structuré de l'enseignant. Dans l'extrait de cours magistral suivant, cette inter-relation apparaît clairement.

Exemple n ${ }^{\circ} 1$ : Extrait du cours magistral sur « le récit autobiographique ", chapitre 2 du DVD d'accompagnement (Mangiante \& Raviez, 2015 : 90). 
Dois-je ou non me perpétuer, je parle pas des enfants qu'on peut avoir, mais me perpétuer par un texte. [...] Est-ce que ça vaut la peine, vous voyez je fais la transition là, il me semble que le récit de vie, c'est un récit de survie, c'est ce que Paul Eluard appelait, très très belle expression, je vais l'écrire : «le dur désir de durer ». C'est un surréaliste donc poète surréaliste [...], alors comment vous comprenez cette jolie phrase ? «Le dur désir de durer » : il est plus simple de ne pas durer, il est plus facile, vu l'état du monde et des choses, de disparaître à jamais. Si on a le désir de durer, c'est, on se pose la question : comment vais-je faire pour durer? Quel est le support? Quel est le moyen [...] pour que les générations futures gardent une trace de moimême? Avec des majuscules bien entendu, bon, ce dur désir de durer fait du récit de vie un récit de survie, on va tenter de survivre dans la conscience collective, on va tenter de survivre dans la mémoire collective.

11 Nous voyons ici comment la référence à Paul Eluard ne peut s'interpréter que par rapport à l'argumentation générale développée par l'enseignant, elle répond en quelque sorte à ses questions, Est-ce que ça vaut la peine...? L'énoncé polyphonique est entrecoupé également de commentaires suspensifs qui assurent l'articulation du raisonnement : vous voyez je fais la transition là...

12 Exemple $\mathbf{n}^{\circ} \mathbf{2}$ : Suite de l'extrait du cours magistral sur « le récit autobiographique »

Bon, par conséquent, cette série de «devenir» est profondément autobiographique : écrire c'est faire son autoportrait, c'est noter des images de soi, c'est écrire des petites notes, c'est faire son journal [...], un vrai faux journal, ce que les surréalistes appelaient, bon, là, je le demandais pas bien entendu, mais c'est une deuxième référence littéraire, "l'explosante fixe ». Je ne crois pas l'avoir déjà expliquée, cette chose-là, ça vient d'André Breton, ça vient de [...], d'André Breton, et il dit au fond la littérature, « c'est réussir à saisir une locomotive qui va à toute vitesse et à la bloquer en quelque sorte dans une image arrêtée ». De ce mouvement gigantesque qui court comme ça, l'avenir vers on sait pas quoi, bon, et il donne un deuxième exemple : « l'explosante fixe, c'est la photographie d'un feu d'artifice » parce qu'un feu d'artifice par essence, c'est du mouvement, de la vie, de l'énergie, voilà, ça éclate, ça fait de la lumière, ça fait de la fumée, ça fait du bruit. Donc un feu d'artifice arrêté, ça a pas de sens, il y a le mouvement de la fusée, le mouvement de l'éclatement, le mouvement qui retombe, la fusée qui retombe, la fumée qui retombe aussi, donc, "l'explosante fixe" serait d'arrêter le feu d'artifice en le prenant en photo, et vous avez donc une saisie, comme ça, immédiate, un flash de ce qu'était le mouvement. Évidemment, c'est une saisie partielle mais, quelque part, peut-être, c'est rassurant dans notre perspective, donc, je vous propose de dire qu'en fait le texte est une « explosante fixe».

Cet extrait illustre le double dialogisme évoqué plus haut, interlocutif avec l'implication nette de l'enseignant qui interpelle son public, le prend à témoin et le sollicite (bon, là, je le demandais pas bien entendu, je vous propose de dire... ; je ne crois pas l'avoir expliqué, et vous avez donc une saisie ...), et interdiscursif avec la convocation de la référence à André Breton (ça vient d'André Breton...).

C'est au travers du dialogisme interlocutif que le discours de l'enseignant entre en relation avec les réactions, attitudes, réponses, interventions des étudiants, exprimées verbalement ou par le silence, les gestes, ou le paraverbal (Bakhtine, 1978: 72). Les marques de ce dialogisme s'insèrent dans le recours aux références présentées par l'enseignant : vous avez donc une saisie..., je vous propose de dire... 
14 La première étape d'une activité de compréhension orale du mécanisme dialogique consistera à demander aux étudiants de relever dans un tableau, pour chaque extrait, les références à des auteurs, comme dans l'activité ci-dessous :

Exemple $n^{\circ} 3$ : Extrait des activités pédagogiques sur le cours magistral consacré au « récit autobiographique "

\begin{tabular}{|l|l|}
\hline Extrait 1 & Extrait 2 \\
\hline C'est ce que Paul & $\begin{array}{l}\text { Ce que les surréalistes appelaient «l'explosante fixe ». Ca vient d'André } \\
\text { Breton et il dit « au fond la littérature, c'est réussir à saisir une } \\
\text { Éluard appelait «le } \\
\text { locomotive qui va à toute vitesse et à la bloquer en quelque sorte dans } \\
\text { une image arrêtée». }\end{array}$ \\
\hline
\end{tabular}

16 Ici l'enseignant de FOU s'attachera à faire repérer les structures introduisant la référence: c'est ce que, ce que les surréalistes appelaient, ça vient de ... Des expressions équivalentes peuvent être apportées également en complément.

17 L'activité suivante explore les fonctions remplies par cette dimension dialogique du discours de l'enseignant. Ces fonctions sont indiquées par le choix des marqueurs d'introduction des références (origine d'une expression : ça vient de... par exemple), par les prises de position à l'égard des théories véhiculées par ces références et les commentaires, hypothèses, reprises de l'enseignant. Les références et les sources peuvent ainsi faire l'objet de reformulations (il me semble que...), de définitions (écrire c'est ...), de précisions ici soulignées par «donc » (donc poète surréaliste, vous avez donc...), ou encore d'explications (il est plus simple de ne pas durer...). Une deuxième écoute plus approfondie des deux extraits du cours magistral conduit les étudiants à rechercher ces fonctions en les dégageant des commentaires de l'enseignant, en soulignant les formes verbales utilisées: Relevez les reformulations, précisions et explications apportées par l'enseignant aux sources citées dans les extraits et soulignez les formes verbales utilisées. Vous utiliserez le tableau suivant. (cf. plus bas). Ces trois types de commentaires sont redéfinis au préalable avec les étudiants.

Exemple $n^{\circ} 4$ : Deuxième extrait des activités pédagogiques sur le cours magistral consacré au « récit autobiographique »

\begin{tabular}{|c|c|c|}
\hline Reformulations & Précisions & Explications \\
\hline $\begin{array}{l}\text { Il me semble que le récit } \\
\text { de vie, c'est un récit de } \\
\text { survie. }\end{array}$ & $\begin{array}{l}\text { le dur désir de durer, C'est un } \\
\text { surréaliste, donc poète } \\
\text { surréaliste }\end{array}$ & $\begin{array}{l}\text { Il est plus simple de ne pas durer, } \\
\text { il est plus facile, vu l'état du monde } \\
\text { et des choses, de disparaître à } \\
\text { jamais. Si on a le désir de durer, on } \\
\text { se pose la question : comment vais- } \\
\text { je faire pour durer? Quel est le } \\
\text { support? Quel est le moyen pour } \\
\text { que les générations futures gardent } \\
\text { une trace de moi-même? }\end{array}$ \\
\hline
\end{tabular}




\begin{tabular}{|c|c|c|}
\hline $\begin{array}{l}\text { Écrire, c'est faire son } \\
\text { autoportrait, c'est noter } \\
\text { des images de soi, c'est } \\
\text { écrire des petites notes, } \\
\text { c'est faire son journal, } \\
\text { un vrai faux journal. }\end{array}$ & $\begin{array}{l}\text { Vous avez donc une saisie } \\
\text { comme ça immédiate, un flash } \\
\text { de ce qu'était le mouvement, } \\
\text { évidemment c'est une saisie } \\
\text { partielle mais quelque part, } \\
\text { peut-être, c'est rassurant dans } \\
\text { notre perspective. }\end{array}$ & $\begin{array}{l}\text { L'explosante fixe, c'est la } \\
\text { photographie d'un feu d'artifice } \\
\text { parce que un feu d'artifice, par } \\
\text { essence, c'est du mouvement, de la } \\
\text { vie, de l'énergie... Voilà, ça éclate, ça } \\
\text { fait de la lumière, ça fait de la } \\
\text { fumée, ça fait du bruit, donc un feu } \\
\text { d'artifice arrêté, ça a pas de sens. }\end{array}$ \\
\hline
\end{tabular}

19 Un cours magistral possède une trajectoire et une cohérence, avec un enchaînement de concepts et de notions mais, dans la réalité, cette trajectoire ne cesse de dévier, en grande partie en fonction des réactions (ou absence de réaction) des étudiants. Ainsi les nombreuses déviations prises par le discours de l'enseignant relèvent de la composante dialogique du cours magistral. Parmi ces déviations qui ponctuent la démonstration, on peut distinguer les parenthèses, sorte de suspensions du cours plus ou moins courtes qui peuvent apporter une précision d'ordre notionnel, pédagogique (une consigne), ou matérielle. Les incises, extra-thématiques ou supra-problématiques, appartiennent, elles, à un ensemble dont l'enseignant suppose que l'étudiant, par son écoute et par ses notes, reconstituera la totalité : le cours est fait d'engrenages que l'enseignant souligne et, durant la même heure, sa parole associe ainsi des éléments qui apparaissent d'abord disjoints (Raviez, 2016 : 154). Enfin les digressions relèvent davantage de l'improvisation et ne constituent pas une transgression du cours mais participent à sa progression, comme s'il y avait, dans la prolifération des références, un sens, non seulement une signification subliminale, mais bel et bien une direction (ibid. : 155).

Ces déviations ponctuent la démonstration, car on ne perdra pas de vue la logique de toute leçon: elle est une argumentation et non une accumulation. Elles relèvent du double-dialogisme défini plus haut et sont repérables à l'oral dans le discours de l'enseignant par des « ellipses éloquentes », voire un silence incitatif : Je ne parlerai pas de... Vous irez vous-mêmes consulter telle grammaire.... Elles sont déclenchées aussi par le jeu des questions-réponses, les réactions du public, l'actualité, ou encore la connexité des thèmes (ibid. : 155).

21 Ce faisant s'élabore une maïeutique où la parole magistrale est sans cesse renvoyée à celui qui la performe en une dynamique de l'échange faite de questions, mais aussi d'attitudes, de regards et de silence (car le silence des étudiants est tout aussi signifiant que leur participation). Toute parole peut entrer dans le cours, s'intégrer dans ses rouages, en renforcer les stratégies et le transformer en chambre d'échos. La parole magistrale n'en reste pas moins un événement, c'est-à-dire une improvisation. Le même cours, en d'autres circonstances, prendra d'autres résonances : il n'est pas plus semblable à luimême que les notes des étudiants ne se ressemblent. Le cours est toujours en deçà de ce qu'il aurait pu être, l'objectif du professeur étant que du début à la fin de l'heure, la polyphonie ne devienne pas cacophonie. En effet l'enseignant craint toujours que cette progression plurielle de son cours n'entraine une difficulté supplémentaire pour la prise de notes des étudiants.

22 La prise de notes, dans ces conditions, paraît difficile pour les étudiants, qui doivent rédiger une transcription fidèle et pertinente d'un discours spiralaire et par nature digressif, sous forme d'un texte écrit linéaire. Les tableaux constituant les activités pédagogiques qui précèdent peuvent alors constituer un cadre, une matrice, pour cette 
transcription. Ils peuvent ainsi illustrer la cohérence et la progression du CM, en ordonnant les catégories d'énoncés à prendre en notes, ici les explications, reformulations et précisions qui constituent la trame et la progression des extraits présentés.

Dans d'autres cas, l'enseignant pourra, par exemple, proposer des grilles de prises de notes à fournir aux étudiants avant le cours, en fonction de la nature du CM : un espace pour les définitions de notions, un espace pour les applications ou les exemples, un espace pour les sources et références, un espace pour les énoncés explicatifs (causes conséquences par exemple). Au lieu de prendre en notes de façon linéaire sans distinction de fonction les différents énoncés du $\mathrm{CM}$, l'étudiant les repère selon leur fonction dans le cours et les place dans les bonnes cases.

\section{Dialogisme, digressions et reconstruction du discours enseignant dans la prise de notes}

24 La difficulté, ou la gageure, consiste à trouver le point d'équilibre : que le discours d'autorité ne s'effiloche pas en parcelles de savoir, que le questionnement du public n'entraîne pas une dérive loin de l'objectif initial. Mais l'on peut aussi voir en la parcelle le fragment d'un tout qui se (re)construit peu à peu, comme en la dérive le mouvement d'une curiosité qui est l'âme de tout apprentissage. On tiendra de même pour certain que si une parole qui s'impose prétend se constituer en modèle, une parole qui se propose et privilégie l'échange peut aussi être un bon moyen d'atteindre le même modèle. Tout cours est un compromis, un modus loquendi, mais aussi audendi. La prise de notes peut donc devenir un exercice de reconstitution du cours pendant ce dernier mais aussi après lui, par des compléments que les étudiants lui apportent par leurs propres recherches et réflexions.

Quels sont donc les échos qui se croisent au fil de l'heure ? L'enseignant est en réalité son premier interlocuteur. Autoréférentiel, son discours progresse en réponse aux questions qu'il a lui-même soulevées, en complément aux nombreux points qu'il a dû éluder, en variations sur des thèmes dont il explore la complexité. On peut citer quelques exemples issus des extraits présentés : Est-ce que ça vaut la peine, vous voyez je fais la transition..., alors comment vous comprenez cette jolie phrase " Le dur désir de durer »? (exemple $n^{\circ} 1$ ).

Le monologue de l'enseignant fait ainsi, si l'on ose dire, boule de neige. Car c'est le monde, c'est la vie même qui sont susceptibles d'entrer dans sa tirade, et plus particulièrement, dans le cadre d'un cours de littérature, tout ce qu'il a lu: l'auteur, la critique, les monographies historiques, études stylistiques, bibliographies ou notes de bas de page qui bourdonnent autour du texte, et tout ce qu'une intertextualité par définition non exhaustive peut apporter de lumières pour expliquer (étymologiquement: déplier) le texte.

27 L'objectif est d'extraire de cette masse de connaissances la quintessence nécessaire à un partage ici et maintenant. Celui-ci évolue selon l'auditoire : le nombre d'étudiants, leurs réactions, leur rapidité à garder trace écrite de ce qui est dit, ainsi que divers facteurs extérieurs, conditionnent l'alliance de méthode et d'intuition qui caractérise l'acte d'enseigner. Gérer tout un passé d'apprentissage en même temps que le présent de la transmission, telle est la gageure. Le cours ne peut en effet être un discours autotélique, qui se refermerait sur lui-même; il ne peut être non plus un émiettement impulsif 
d'éléments disparates. On insistera ici sur le pouvoir de l'émotion, qui motive la parole tout autant que les impératifs didactiques ou le respect du programme. Le cours est la chambre d'échos du savoir et de l'expérience, une sélection en temps réel d'éléments susceptibles de se constituer en argumentaire, c'est-à-dire en une progression équilibrée d'interprétations. Celle-ci suppose une longue incubation, mais surtout une tension constante du corps et de l'esprit. Parler ex cathedra pendant une heure ou plus convoque tout ce que je sais, mais aussi tout ce que je suis, et, quelle que soit mon humeur, m'oblige à me donner en spectacle.

Le travail de remémoration, traversé de tout ce qui passe dans l'esprit à l'énoncé d'un nom, d'un titre ou d'une date (c'est ce que Paul Eluard appelait, très très belle expression, je vais l'écrire, exemple $n^{\circ} 1$ ), mais aussi le travail d'oralisation en termes clairs, sur un rythme adapté à l'auditoire, d'un cours préalablement fixé sur le papier, enfin la gestion des incidents et incidences de l'instant, tout cela impose ce que l'on pourrait appeler une urgence maîtrisée à quoi l'art du professeur s'emploie à donner l'apparence du naturel. Magister dixit, certes, mais à quel prix ? Celui d'un monologisme tenté par l'interactivité, d'une structure herméneutique menacée par la digression, d'une copia qui redoute l'extinction... Que dire ? Telle est la question. Mais aussi : que doivent-ils savoir ? Par quel effet d'oralisation, par quelle spontanéité professionnelle, par quelle amorce artificielle de dialogue dois-je passer pour transmettre un écho crédible de tout ce qui parle en moi ? Comme il a travaillé son auteur, le professeur travaille son charisme, en prenant le mot au sens large: son autorité, son influence, sa rhétorique, sa méthode, son verbe, son humanité.

Que ce soit dans les digressions, dans les échanges avec les étudiants, les commentaires sur les références, les explications et reformulations, l'enseignant alterne donc les partis pris personnels par rapport aux discours des autres auxquels ils se réfèrent, et les données objectives qui fixent son cadre théorique. En tant qu'expert de sa discipline, il décrit, expose, raisonne, avec la distance scientifique qu'exige la situation... l'enseignant universitaire est aussi un chercheur critique, c'est également un pédagogue soucieux de se rapprocher de ses étudiants... (Mangiante \& Parpette, 2011 : 67).

Cette implication peut surprendre et perturber les étudiants, particulièrement les étudiants allophones dont la culture universitaire est différente dans leur pays d'origine. Il conviendra donc de les préparer à distinguer les différentes attitudes de l'enseignant et à en comprendre la fonction pédagogique, notamment dans une prise de notes qui tient compte de leur rôle dans l'organisation générale du cours.

31 L'extrait de cours magistral qui suit illustre cette particularité du discours enseignant.

32 Exemple $n^{\circ} 5$ : Extrait du cours magistral consacré aux Mémoires d'outre-tombe de Chateaubriand, chapitre 2 du DVD d'accompagnement (Mangiante \& Raviez, 2015 : 87).

\footnotetext{
Alors nous étions devant un sujet, là, qui, un énorme sujet avec un énorme plan, donc je vais devoir tout de suite shunter ce petit commentaire. Sujet pas facile dont nous n'avons fait que le grand 1 en $1 \mathrm{~h} \mathrm{30}$, donc, comme je suis long à le terminer, je crois qu'il est grand temps d'y revenir. Alors, on a déjà traité finalement ce sujet, plus ou moins, lorsqu'on a parlé de cette petite phrase, là, à la fin de la quatrième partie [...]. Dans une lettre de 1800 à un de ses proches Chateaubriand présente ainsi le projet des Mémoires d'outre-tombe: "soyez tranquilles », non je lis mal: "soyez tranquilles, ce ne seront point des confessions pénibles pour mes amis "; là il prend la pose,
} 
«si je suis quelque chose dans l'avenir... ». Donc on imagine les amis autour disant "quel grand homme!", "les amis y liront un nom aussi beau que respectable, je n'entretiendrai pas non plus la postérité des détails de mes faiblesses, je ne dirai de moi que ce qui est convenable à ma dignité d'homme et j'ose le dire à l'élévation de mon cœur ». Insupportable ce personnage, ça, c'est vraiment l'autoglorification, et de conclure : «il ne faut présenter au monde que ce qui est beau ce n'est pas mentir à Dieu que de ne découvrir de sa vie que ce qui peut porter nos pareils à des sentiments nobles et généreux ». J'ajouterai volontiers : amen.

Cet extrait est représentatif du dialogisme interlocutif à fonction pédagogique du discours enseignant dans sa volonté de connivence avec les étudiants (sujet pas facile dont nous n'avons fait que le grand 1...). Le commentaire de l'enseignant s'inscrit aussi dans une forme de digression dont il souligne la portée et l'importance pour son raisonnement: donc je vais devoir tout de suite shunter ce petit commentaire; comme je suis long à le terminer, je crois qu'il est grand temps d'y revenir.

Le discours alterne également les énoncés objectifs fixant les principes à retenir : dans une lettre de 1800 à un de ses proches, Chateaubriand présente ainsi le projet des Mémoires d'outretombe... qui est aussi une référence bibliographique, et les commentaires personnels, prises de position très critiques: là il prend la pose, Insupportable ce personnage, ça c'est vraiment l'autoglorification, j'ajouterai volontiers : amen.

Cet extrait s'inscrit bien également dans ce double dialogisme, interlocutif appliqué par l'enseignant pour tenir compte des réactions des étudiants, anticiper sur leur appréciation des commentaires et des notions à comprendre, et se rapprocher d'eux, et interdiscursif avec la référence à Chateaubriand.

36 Par les activités suivantes, les étudiants du cours de FOU doivent se préparer à une prise de notes efficace en repérant les énoncés objectifs, les prises de position scientifiques et les attitudes dialogiques.

7 Ils doivent écouter l'extrait et remplir un tableau distinguant les différents énoncés de l'enseignant.

38 Exemple n ${ }^{\circ} 6$ : Extrait des activités pédagogiques sur le cours magistral consacré aux Mémoires d'outre-tombe de Chateaubriand, chapitre 2 du DVD d'accompagnement (Mangiante \& Raviez, 2015 : 87 et 88).

\begin{tabular}{|c|c|}
\hline Phrases présentant des données objectives & $\begin{array}{l}\text { Phrases exprimant une opinion, un } \\
\text { avis }\end{array}$ \\
\hline $\begin{array}{l}\text { Dans une lettre de } 1800 \text { à un de ses proches, } \\
\text { Chateaubriand présente ainsi le projet des Mémoires } \\
\text { d'outre-tombe: } \\
\text { "soyez tranquille, ce ne seront point des } \\
\text { confessions pénibles pour mes amis» }\end{array}$ & $\begin{array}{l}\text { Là, il prend la pose: «si je suis quelque } \\
\text { chose dans l'avenir ", donc on imagine } \\
\text { les amis autour disant «quel grand } \\
\text { homme!» }\end{array}$ \\
\hline $\begin{array}{l}\text { "Je n'entretiendrai pas non plus la postérité des } \\
\text { détails de mes faiblesses, je ne dirai de moi que ce } \\
\text { qui est convenable à ma dignité d'homme et j'ose le } \\
\text { dire à l'élévation de mon cœur" }\end{array}$ & $\begin{array}{l}\text { Insupportable ce personnage, ça c'est } \\
\text { vraiment l'autoglorification }\end{array}$ \\
\hline $\begin{array}{l}\text { Et de conclure : «Il ne faut présenter au monde que } \\
\text { ce qui est beau» }\end{array}$ & $\begin{array}{l}\text { Sentiments nobles et généreux. } \\
\text { J'ajouterai volontiers: amen. }\end{array}$ \\
\hline
\end{tabular}


Là encore l'activité de repérage doit être assortie d'une mise en évidence des structures verbales utilisées par l'enseignant pour permettre une meilleure prise de notes, au cours d'une deuxième écoute approfondie de vérification et de correction du premier tableau.

Dans l'exemple suivant les étudiants doivent reprendre les phrases exprimant des données objectives et relever les verbes utilisés par l'enseignant.

Exemple $n^{\circ} 7$ : Extrait des activités pédagogiques sur le cours magistral consacré aux Mémoires d'Outre-tombe de Chateaubriand, chapitre 2 du DVD d'accompagnement (Mangiante \& Raviez, 2015 : 87 et 88).

\begin{tabular}{|c|c|}
\hline Phrases & Structures verbales \\
\hline $\begin{array}{l}\text { "Chateaubriand présente ainsi le projet des Mémoires d'Outre- } \\
\text { tombe" }\end{array}$ & $\begin{array}{l}\text { Présenter (présent de } \\
\text { l'indicatif). }\end{array}$ \\
\hline $\begin{array}{l}\text { "On a déjà traité finalement ce sujet plus ou moins lorsqu'on a } \\
\text { parlé de cette petite phrase, là, à la fin de la quatrième partie " }\end{array}$ & $\begin{array}{l}\text { Traiter un sujet (passé } \\
\text { composé) } \\
\text { Parler de (passé composé). }\end{array}$ \\
\hline $\begin{array}{l}\text { "Soyez tranquille, ce ne seront point des confessions pénibles } \\
\text { pour mes amis" }\end{array}$ & $\begin{array}{l}\text { Être (impératif présent) } \\
\text { Être pour (futur simple de } \\
\text { l'indicatif) }\end{array}$ \\
\hline $\begin{array}{l}\text { "Je n'entretiendrai pas non plus la postérité des détails de mes } \\
\text { faiblesses, je ne dirai de moi que ce qui est convenable à ma } \\
\text { dignité d'homme et j'ose le dire à l'élévation de mon cœur » }\end{array}$ & $\begin{array}{l}\text { Entretenir la postérité de } \\
\text { (futur simple de } \\
\text { l'indicatif) } \\
\text { Dire de (futur simple de } \\
\text { l'indicatif) } \\
\text { Être (présent de } \\
\text { l'indicatif) } \\
\text { Oser + verbe à l'infinitif } \\
\text { (présent de l'indicatif - } \\
\text { concordance des temps) }\end{array}$ \\
\hline «Il ne faut présenter au monde que ce qui est beau» & $\begin{array}{l}\text { Falloir + verbe à l'infinitif } \\
\text { (présent de l'indicatif) } \\
\text { Être (présent de } \\
\text { l'indicatif). }\end{array}$ \\
\hline
\end{tabular}

41 Les différentes structures verbales à repérer sont représentatives de ce discours à soimême qu'est en partie un cours, dont tout l'art de l'enseignant a consisté à lui donner la forme et la force de l'adresse. Les procédés, comme on le voit ici, en sont multiples, qui appartiennent à la fois à l'art oratoire et à la conversation. On ne saurait ici recenser tout ce qui ressort de l'éloquence antique, voire de l'art dramatique, dans la manière de capter, captiver un auditoire, par exemple l'apostrophe, la question rhétorique, la phrase interro-négative, l'impératif, mais aussi la voix et la gestuelle. Tous illustrent le dialogisme interlocutif propre au cours magistral. De la conversation, on retiendra le goût d'un échange dirigé, avec les étudiants, une spontanéité intéressée, l'attention à l'autre, sans oublier la tendance au coq-à-l'âne, l'allusion ou l'ironie (J'ajouterai volontiers: amen). Le maître est maître du jeu (et du Je); il peut lancer et interrompre l'échange, le faire dévier, l'orienter et le conduire après quelques détours là où il voulait arriver. Il donne 
l'illusion que la leçon se conduit avec l'aide des «apprenants». Certes, il est des conversations impossibles (le cours magistral), et d'autres où la liberté du dire compte moins que les objectifs (les travaux " dirigés »). S'il y a imposture, elle sert à apprendre, car elle adoucit ce que la tirade magistrale pourrait avoir d'indigeste.

On se souvient, parmi les mythes pédagogiques de ces dernières décennies, de la «participation», promue condition sine qua non d'un «bon» cours. Il va de soi que personne ne souhaite avoir devant soi pendant de longues heures des étudiants muets comme des carpes, mais comment traiter de la complexité d'une œuvre ou des ambiguïtés d'un texte par rebond néo-convivial, stimuli pseudo-formateurs, maïeutique du vide pour créer du sens?

L'échange, au sein de la classe, est biaisé par les horaires, les programmes et surtout les notes. Il est traversé de stratégies de séduction, mais aussi d'évitement, et " frémit » de diverses angoisses. Le cours doit en tenir compte, comme il ne peut faire l'économie de la vie réelle, des contingences du présent à la pression de l'histoire. Comment reprendre la parole après des événements traumatiques ? La classe n'est pas un monde lointain, isolé $\mathrm{du}$ bruit du monde. L'histoire s'y engouffre et le travaille; la chambre d'échos se confronte au vacarme du quotidien. Comment gérer ce moment du présent qu'est la leçon tout en préservant une distance scientifique, une objectivité relative, une chimérique intemporalité ? Dans ce compromis qu'est le cours, la parole est la longue modulation d'un savoir au risque des choses et des individus.

Moduler, c'est transformer, absorber pour mieux déployer, limiter tout en ouvrant le champ des significations, faire la part du topos et celle d'un vocabulaire spécifique; moduler, c'est s'employer à tenir un discours médian : ni celui de la rue, ni celui du savant.

Les dialogues qui s'entrecroisent au sein d'un cours sont nécessairement hybrides, d'abord parce que la parole « pure » n'existe pas ; ensuite parce que tout discours adressé, s'il veut perdurer, doit tenir compte des capacités de réception de son destinataire ; enfin parce que sa dynamique rend cette parole, selon les occurrences, simple et complexe, familière et scientifique, collective et individuelle. La modulation se modèle sur un public: s'il est aujourd'hui exotique d'employer un imparfait du subjonctif, son usage systématique finirait par passer pour une revendication de différence. De même, un ton trop familier, haché d'interruptions ou d'interjections d'attente, dévalorise le message. Trop de noms propres inconnus, de références à des œuvres que personne n'a lues, trop d'implicite, mais aussi un recours abusif à ces termes à consonances grecques (anacéphaléose, paryponoïan...) dont l'effet quasi magique est assuré, trop de science, en un mot, finit par étouffer la science. Inversement, une simplification systématique écraserait toute nuance au profit d'un discours démagogique, et finirait par diluer la parole magistrale dans le banal, l'insignifiant.

Trouver le ton, tout est là. La difficulté est que ce ton ne peut être entièrement prémédité, car, on l'a vu, en condition de cours, l'improvisation induit une parlure sur le vif, une captatio aux ruses multiples (Raviez, 2016: 158). Il faut occuper l'heure, en garder la maîtrise du début à la fin, dominer son sujet, et sa classe, et soi-même, et répercuter ce pouvoir dans chaque syntagme de son dire (un ton d'autorité n'est pas toujours magistral), dans son rapport au groupe et à chacun, dans une présence que rien, pour qu'elle reste crédible, ne peut, ne doit déconsidérer ou interrompre, sous peine de voir la fiction pédagogique s'évaporer. Le maître, pour employer une expression du Grand Siècle, 
se fait « tout à tous ", tout à son discours (fabriqué, on l'a vu, de plusieurs composantes), tout aux bribes de l'échange et à la continuité de sa démonstration. D'une heure à l'autre, d'un public à l'autre, d'un lieu à l'autre, les cartes sont rebattues, le ton change, un nouveau dialogue s'instaure. La parfaite ductilité de la langue permet les variations d'une adresse sans cesse renouvelée, par conséquent d'une relation au groupe qui n'est jamais exactement reproductible.

\section{En conclusion}

Le concept de dialogisme, tel que Bakhtine l'a défini, constitue un phénomène d'intertextualité, par lequel des textes interagissent en contexte soit par le dialogue qui s'instaure entre les locuteurs, on parle alors de dialogisme externe, soit à l'intérieur d'un texte, un cours magistral par exemple, où toute parole renvoie à d'autres sources, références ou auteurs, de façon explicite (polyphonie) ou implicite, on parle alors de dialogisme interne. Le principe directeur de ce dialogisme est qu'il n'est pas un seul énoncé verbal qui puisse, en quelque circonstance que ce soit, être porté au seul compte de son auteur [...] (Bakhtine \& Volochinov, 1980). La compréhension d'un texte, en particulier ici d'un cours magistral, dépend donc de la perception du réseau d'interrelations dans lequel il se situe et se détermine. Ainsi le cours magistral apparait monologique dans sa forme extérieure mais sa structure stylistique et sémantique est essentiellement dialogique (Volochinov, 1981 : 292, cité par Charaudeau \& Maingueneau, $2002: 175$ ). Quand l'enseignant prend la parole, il se positionne en situation de dialogue et son discours est caractérisé par l'altérité. Le concept bakhtinien du dialogisme remet en cause non seulement l'unicité de l'énoncé, mais également celle du sujet parlant (Calabrese-Steimberg, 2010 : 60-64).

Ainsi la parole magistrale est un lieu d'interférences, et le cours une fiction où chacun s'emploie à tenir sa partie. Rien de péjoratif dans ce mot de fiction, mais au contraire une invention renouvelée au quotidien, une intrigue herméneutique à laquelle chacun peut participer à sa manière, une histoire collective. Même le cours magistral induit une interactivité inversement proportionnelle au nombre d'étudiants : consubstantielle de la leçon en petit groupe, mais, il faut bien le reconnaître, plutôt factice en amphithéâtre. Si le dialogue du professeur avec les grandes figures qu'il cite et ressuscite reste un échange à sens unique, un semi-dialogue des morts, celui avec les vivants tient en deux mots : l'attention et l'intention. On n'imagine pas un cours neutre ou indifférent, hors de tout contexte. Cependant le point d'équilibre évoqué plus haut ne se découvre pas à la minute où le professeur paraît devant sa classe : il se travaille, s'expérimente et se remet en cause dans le progrès même de la leçon. Évolutif comme tout savoir, mouvant comme toute relation, il est le produit d'une expérience, d'un savoir et d'une confiance. L'expérience s'est forgée, fortifiée au fil du temps ; le savoir se doit d'être incontestable. Quant à l'ethos du professeur, à lui de le tisser mot après mot, dans un échange un peu faux, un peu forcé, mais riche de vérités. 


\section{BIBLIOGRAPHIE}

Bakhtine, M. (1938/1978 version française). Esthétique et théorie du roman. Paris : Gallimard.

Bakhtine, M. \& Volochinov, V. N. (1980). Le Freudisme. Lausanne : L'Age d'Homme.

Calabrese-Steimberg, L. (2010). «Esthétique et théorie du roman : la théorie dialogique du Bakhtine linguiste ", Slavica bruxellensia. Revue polyphonique de littérature, culture et histoire slave, $\mathrm{n}^{\circ} 6$. Linguistique russe.

Charaudeau, P. \& Maingueneau, D. (2002). Dictionnaire d'Analyse de discours. Paris : Seuil.

Mangiante, J.-M. \& Parpette, C. (2011). Le Français sur Objectif Universitaire. Grenoble : PUG.

Mangiante, J.-M. \& Raviez, F. (2015). Réussir ses études littéraires en français. Grenoble : PUG.

Raviez, F. (2016). « La (dé)composition du cours ou les paradoxes de l'enseignant ». In Bordo, W., Goes, J. et Mangiante, J.-M. (dir.). Le Français sur Objectif Universitaire. Entre apports théoriques et pratiques de terrain. Arras : APU.

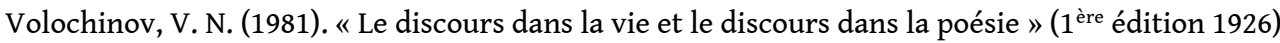
et « la Structure de l'énoncé » (1 ère édition 1930). In Todorov, T. Mikhail Bakhtine. Le principe dialogique. Paris : Seuil.

\section{RÉSUMÉS}

Le cours magistral de littérature dans les filières de Licence de Lettres de l'enseignement supérieur français, manifeste un dialogisme caractérisé par des formes digressives et polyphoniques. Cette propension à la digression ne relève pas d'un détournement rhétorique de la parole enseignante vers des énoncés latéraux secondaires, voire facultatifs, dans le raisonnement de l'enseignant, mais concourt véritablement à la compréhension du cours et au dialogisme de sa forme. Cet article se propose de définir et de décrire le dialogisme particulier du cours magistral de littérature en le reliant à la polyphonie des discours convoqués par l'enseignant. Il s'appuiera sur le corpus de cours magistraux constitués par le laboratoire Grammatica de l'université d'Artois dans le cadre de son programme de recherche sur le Français sur Objectif Universitaire.

The lecture of literature in the Bachelor of Arts in French higher education, shows a dialogism characterized by digressive and polyphonic forms. This propensity to digression does not depend on a rhetorical diversion of the teaching speech towards secondary or even facultative lateral utterances in the reasoning of the teacher but truly contributes to the understanding of the course and the dialogism of its form. This article proposes to define and describe the particular dialogism of the lecture of literature by linking it to the polyphony of the discourses summoned by the teacher. It will be based on the corpus of lectures organized by the Grammatica laboratory of the University of Artois as part of its research program on the French for Academic Purposes. 
INDEX

Mots-clés : dialogisme, polyphonie, digressions, cours magistral

Keywords : dialogism, polyphony, digressions, lectures

\section{AUTEURS}

FRANÇOIS RAVIEZ

Université d'Artois - Laboratoire Textes et Cultures

François RAVIEZ, ancien élève de l'École Normale Supérieure de Saint-Cloud, agrégé de Lettres modernes, est maître de conférences habilité en Langue et littérature françaises. Spécialiste du genre des Mémoires et de l'autobiographie, il enseigne principalement la littérature d'Ancien Régime et de la Restauration de la première année de Licence à l'agrégation interne, ainsi que la stylistique en Master d'enseignement. Ses recherches portent sur l'écriture de soi, la poétique de l'histoire et le spirituel.

Francois.raviez [at] univ-artois.fr

\section{JEAN-MARC MANGIANTE}

Université d'Artois - Laboratoire Grammatica

Jean-Marc MANGIANTE est Professeur des Universités en Linguistique française et Didactique du FLE, responsable du Master FLE/FLS/FOS en milieux scolaire et entrepreneurial de l'université d'Artois, directeur du DU de français sur objectif universitaire pour la préparation des étudiants allophones à l'enseignement supérieur. Il a été directeur du centre de langue française de la chambre de commerce et d'industrie de Paris et directeur adjoint de l'institut français de Rabat. Jmarc.mangiante [at] univ-artois.fr 